\title{
Additive Effect of Carbon Nanostructures on the Microstructures and Properties of Pitch Derived Carbon Foam Composites
}

muhammad khan ( $\triangle$ mkhanchemistry@yahoo.com )

Northwestern Polytechnical University https://orcid.org/0000-0001-6883-1394

amjad hussain

Department of Chemistry University of Okara

hammad Ahmad

Department of Chemistry University of Okara

Li Tiehu

Northwestern Polytechnical University

Amir Zada

Abdul Wali Khan University

hidayat hussain

Leibniz Institute of Plant Biochemistry

javid hussain

University of Nizwa

muhammad imran

King Khalid University

ataf ali altaf

Department of Chemistry University of Okara

\section{Research Article}

Keywords: Carbon foam, carbon nanotubes, nanodiamonds, microstructure, mechanical and electrical properties

Posted Date: March 12th, 2021

DOl: https://doi.org/10.21203/rs.3.rs-277708/v1

License: (c) (i) This work is licensed under a Creative Commons Attribution 4.0 International License.

Read Full License 


\section{Abstract}

In the present work, carbon foam (CF) coupled multi-wall carbon nanotubes (MWCNTs) and CF/MWCNTs coupled nanodiamond (NDs) hybrid composites were prepared by the direct treatment of MWCNTs and NDs with pretreatment pitches through foaming, graphitization, and carbonization process. Both series of

CF composites were compared and explored by Fourier-transform infrared spectroscopy, X-ray diffraction, scanning electron microscopy and energy dispersive X-ray spectroscopy techniques. It was investigated that the structural morphology, pore size and pore uniformity upgraded with the increment of MWCNTs content, however, the effect of ND particles in this aspect was more influential. Furthermore, the effect of additive loading of MWCNTs and NDs on the mechanical, electrical and thermal properties of CF composites were checked. The results revealed that maximum compressive strength $15.8 \mathrm{MPa}$ and electrical conductivity $30.2 \times 10^{3} \mathrm{~S} / \mathrm{m}$ of CFs were obtained at $4 \mathrm{wt} . \%$ MWCNTs and ND loadings respectively. While the maximum thermal conductivity value of $32.2 \mathrm{Wm}^{-1} \mathrm{k}^{-1}$ was revealed by $\mathrm{CFs}$ having 2 wt.\% MWCNTs and NDs loadings.

\section{Introduction}

Carbon foam (CF), a sponge like substance with distinct characteristics such as low density, large external surface area, excellent thermal strength and corrosive resistivity has versatile characteristics and significant consideration in the recent past ${ }^{[1-4]}$. On the basis of these properties, CFs are considered as appropriate material for many industrial applications such as, energy storage devices (super capacitors), electrode material for electrical double-layered capacitors, energy storage material, catalyst supporting material, electrode material for fuel cell, filtering material, electromagnetic shielding, bone-surgery applications and gas filtration ${ }^{[2-5-8]}$. Since the CF obtained from various sources such as organic polymer, coal tar, petroleum pitches and synthetic pitches have limited thermal and electrical conductivities as well as low mechanical properties, therefore, such type of $\mathrm{CF}$ is not suitable for many applications and these properties are under huge investigation during the last few decades ${ }^{[5-9-11]}$. In order to improve the mechanical and thermal properties of $\mathrm{CF}$, many additives and reinforcing methods like hollow ceramic microsphere have been set in CFs. Also, the progress and applications of CF are seriously constrained due to its poor mechanical characteristics and large manufacturing charge ${ }^{[13]}$. Wang et al. obtained CF from the thermal treatment of coal tar-based pitches reinforced with clay with much improved mechanical characteristics. However, the thermal conductivity were decreased from 2 to $0.252 \mathrm{Wm}^{-1} \mathrm{~K}^{-1}$ [26]. Similarly, although CF derived from various resins has low thermal conductivity and is better for high temperature insulation, the mechanical properties of these resins-derived CF are not encouraging. Therefore, much attention is needed to investigate the mechanical properties of CF.

A high pressure method (complex process) has been applied most frequently to enhance the mechanical properties of CFs. For this purpose, CF derived from resins, coals or biomass materials were made-up by stabilizing and carbonizing. The disadvantage of this process is that during carbonization, CF undergoes disintegration resulting in structural defects and hence reduces the mechanical properties of CF. The 
mechanical strength can be enhanced by reducing structural cracks ${ }^{[3-6-14]}$. The Beechem and colleagues, introduced a novel high strength CF through the integration of carbon nanofibers into a pitch based precursor, which improved the compressive strength to $6 \mathrm{MPa}{ }^{[10]}$. Li et al produced CF with high compressive strength by mixing mesocarbon microbeads and mesophase pitch. By this method, the highest compressive strength of about $23.7 \mathrm{MPa}$ was achieved at bulk density of $0.78 \mathrm{~g} / \mathrm{cm}^{3}$, but thermal conductivity of the material decreased with the increasing amount of mesocarbon microbeads ${ }^{[12]}$. In recent studies, some other materials such as $\mathrm{K}_{2} \mathrm{Ti}_{6} \mathrm{O}_{13}, \mathrm{SiO}_{2}$ aero gel have been reported as additive in $\mathrm{CF}$ to improve the mechanical and thermal properties of $\mathrm{CF}$ composites ${ }^{[16-17]}$. The multi walled carbon nanotubes (MWCNTs) and nanodiamonds (ND) are pure carbon nanomaterials and they are strongly considered as ideal and advance nanofiller materials in the recent carbon-carbon nanocomposites [3-7-14]. The mechanical and thermal properties of MWCNTS and ND particles are much better than that of the other nanomaterials ${ }^{[3-15]}$.

In this study, the mechanical, thermal and electrical properties of carbon foam composites reinforced by MWCNTs and hybrid MWCNTs-ND were studied and reported by varying the reinforcement amounts. The various contents of MWCNTs and NDs were taken and then directly treated with mesophase pitches for the preparation of CF composites and finally their mechanical, thermal and electrical performances were checked and compared.

\section{Experimental Part}

\section{Materials}

Coal tar pitches were acquired from Wuhan Steel Company Limited China for the production of CF. Some physical properties of local coal tar pitches are given below in table-1. The ND powders were received from Hengqiu Nanotechnology Corporation China, having dark gray color with $99.5 \%$ purity. The particle size of ND was $5 \mathrm{~nm}$ and particle size distribution 5-10 nm. MWCNTs having purity of $95.2 \%$ were obtained from well-known Tsinghua University China. The obtained MWCNTs were further modified and purified with concentrated $\mathrm{HNO}_{3}$ (Xi'an Sanpu Fine Chemical Factory, Xi'an, China) and concentrated $\mathrm{H}_{2} \mathrm{SO}_{4}$ (Beijing Chemical Works, Beijing, China). Both these chemicals were preferred as a reaction reagent for the purification and modification.

Table.1 physical characteristics of commercial coal tar pitch.

\begin{tabular}{|lcccc|}
\hline $\begin{array}{l}\text { Soft point } \\
\left({ }^{\circ} \mathrm{C}\right)\end{array}$ & $\begin{array}{c}\text { Quinoline insoluble } \\
(\text { wt.\%) }\end{array}$ & $\begin{array}{c}\text { Benzene insoluble } \\
(\text { wt.\%) }\end{array}$ & $\begin{array}{c}\text { C/H (atomic) } \\
(\text { ratio })\end{array}$ & $\begin{array}{c}\text { Carbon yield } \\
\text { (wt.\%) }\end{array}$ \\
\hline 81 & 6.55 & 20.3 & 1.72 & 55.1 \\
\hline
\end{tabular}

\subsection{Purification of NDs}


Heat treatment method was used for the purification of NDs. In this method, oxidation of the ND particles was carried out in the presence of air at $440^{\circ} \mathrm{C}$ in electric kiln to eliminate the impurities such as metal oxides and other volatile impurities probably added during the production of ND particles. In this method, ND particles remained unchanged at elevated temperature while impurities such as metal oxides and non-carbonaceous materials are burnt out and removed.

\subsection{Surface Functionalization of ND powders}

Functionalization is strongly decreases aggregation of NDs because ND particles have large surface energy owing to small size and huge surface to volume ratio ${ }^{[10]}$. After heat treatment method, NDs were further exposed to functionalization with $\mathrm{NH}_{2}$ group while the de-aggregation of $\mathrm{ND}$ particles has been achieved through ball milling method. Oxidized ND particles were mixed with $\mathrm{NH}_{4} \mathrm{HCO}_{3}$ in the presence of $\mathrm{NaCl}$ for de-aggregation with a ratio of 1:4. The mixture was placed on miller for two hours with a speed of 500 revolutions per min along with stainless steel ball with diameter of $2 \mathrm{~mm}, 3 \mathrm{~mm}$, and $5 \mathrm{~mm}$. In order to remove humidity, the samples were set in vacuum furnace at $0.7 \mathrm{MPa}$ for 10 hours at $100^{\circ} \mathrm{C}$. During the process, $\mathrm{NH}_{4} \mathrm{HCO}_{3}$ is detached and amines groups are attracted to the surface of ND particles through covalent bonds according to the following equation ${ }^{[18]}$.

$$
2 \mathrm{NDs}+2 \mathrm{NH}_{4} \mathrm{HCO}_{3} \longrightarrow 2 \mathrm{ND}-\mathrm{NH}_{2}+2 \mathrm{CO}_{2}+3 \mathrm{H}_{2} \mathrm{O} \quad \mathrm{Eq}(1)
$$

\subsection{Purification of MWCNTs}

For the purification of MWCNTs, a mixture of conc. nitric acid and sulfuric acid (1:3) was prepared and MWCNTs were dispersed in it. As result, a semi liquid mixture (slurry) appeared. The mixture was agitated at $65^{\circ} \mathrm{C}$ for seven hours and washed with deionized water several times. The MWCNTs collected on filter paper placed in Buchner funnel were dried at $85^{\circ} \mathrm{C}$ for several hours.

\subsection{Preparation of carbon foam (CF) composites}

The pretreated pitches were synthesized from commercial coal tar pitch. First of all, $120 \mathrm{~g}$ of coal tar pitch were finely grounded to $72 \mu \mathrm{m}$ and then heated under the nitrogen atmosphere at $425^{\circ} \mathrm{C}$ for 6 hours with a heating rate of $1.5^{\circ} \mathrm{C} / \mathrm{min}$. For the production of $\mathrm{CFs}$, the obtained pretreated pitches were again finely grounded and mixed with different amounts of MWCNTs and hybrid MWCNTs-NDs composites ${ }^{\text {[32] }}$. In order to obtain high quality and uniform dispersion of different wt.\% of the nanofillers i.e. MWCNTs and its hybrid MWCNTs-ND, the pretreated pitches were treated with $\mathrm{C}_{2} \mathrm{H}_{5} \mathrm{OH}$ solution because the uniform dispersion of reinforcements into matrix usually determines the characteristics features of the composites. The mixtures of pretreated pitches along with reinforcements were heated at $90{ }^{\circ} \mathrm{C}$ in order to remove $\mathrm{C}_{2} \mathrm{H}_{5} \mathrm{OH}^{[32]}$. The final mixture was ground to $\sim 72 \mu \mathrm{m}$. Finally, the foaming process of MWCNTs/pretreated pitches and MWCNTs-NDs/pretreated pitches were followed in a reaction vessel containing $\mathrm{N}_{2}$ atmosphere maintained at $3 \mathrm{MPa}$. First, the mixture was heated at $600^{\circ} \mathrm{C}$ for 2 hours with 
a heating rate of $1.5^{\circ} \mathrm{C} / \mathrm{min}$. The obtained samples were graphitized and carbonized at 800 and $2400{ }^{\circ} \mathrm{C}$ in the presence of $\mathrm{N}_{2}$ atmosphere for 180 and 90 min respectively with a heating rate of $2{ }^{\circ} \mathrm{C} / \mathrm{min}$. Finally, by this way two series of CF composites, (1,2 and 4 wt.\% additive amount of MWCNTs as well as hybrid MWCNTs-NDs) were prepared and further investigated for various characterizations were conducted.

\subsection{Characterization}

Transmission electron microscopy (TEM Hitachi H-600) was used to investigate the morphology and microstructure of CF containing purified MWCNTs and functionalized NDs. X-ray diffraction (XRD) with $\mathrm{Cu}$-Ka radiation analysis was performed to investigate the structural information of the samples. The XRD models of CFs were obtained in the scanning range of $20-80^{\circ}$ in a step size manner using $0.002^{\circ}$ each time. In order to confirm the connection of amines group on the surface of NDs, Fourier transformed infra-red spectrometer (FTIR) was used. Mechanical properties such as compressive power of samples were specified at room temperature by means of universal testing machine with cross head speed of 5 $\mathrm{mm} \mathrm{min}{ }^{-1}$. For this purpose, sample of $20 \mathrm{~mm}$ in height and $15 \mathrm{~mm}$ in diameter was compressed between two stainless plates. The compressive yield strength ( was calculated from the following equation.

$$
\sigma=\frac{\mathrm{F}}{\mathrm{A}}
$$

Here $\mathrm{F}$ is load at yield and $\mathrm{A}$ is the cross sectional area.

\section{Results And Discussion}

\subsection{Transmission electron microscopic (TEM) measurements}

Transmission electron microscopy (TEM) was used to confirm the particle size in addition to average range. Fig. 1(a) and (b) show the TEM images of non-purified and purified MWCNTs whereas (c) and (d) designate the TEM images of non-functionalized and functionalized NDs respectively. Fig. 1(a) indicates that both MWCNT and ND are in nm range ${ }^{[29-32]}$. It is clear from TEM image in 1(a) MWCNTs have several agglomerations on the surface of non-purified samples before the purification due to presence of impurities. After surface modification (purification) by means of conc. nitric acid and sulphuric acid, the tendency of agglomeration was considerably decreased. TEM image in 1(c) also indicates several agglomeration on the surface of non-purified NDs ${ }^{[29]}$. After surface treatment, agglomeration among ND particles were reduced and continuous arrangements were observed in TEM images.

\subsection{X-ray differation (XRD) analysis}

The crystalline structure of carbon foams containing different additives were checked by XRD analysis. Fig. 2 presents the XRD patteren of carbon foams reinforced by ND particles and MWCNTs. It can be observed that the diffraction peaks at $2 \theta=26.1,43.1,54.2$ and $78.3^{\circ}$ which are indexed to the interlayer spacing (002), (101), (004) and (110) respectively show the existence of CF with graphitic crystallite and 
microstructure ${ }^{[10-22]}$. Although CF composites carrying ND particles and MWCNTs as additive acquire one more peak at $2 \theta=75.1^{\circ}$ analogous to (220), entirely fulfilled the presence of cubic structure of Diamond lattice at $\mathrm{nm}$ scale. The XRD analysis of CF composites containing various amount of ND particles and MWCNTs indicates that all the characteristic peaks of CF are appeared on their subsequent point, while the new peak at $2 \theta=75.1^{\circ}$ which indicates the presence of nano particles further verifies the enhancement of crystalline structure of carbon foams ${ }^{[15]}$.

\subsection{Scanning electron microscopic study of CF composites}

The microstructures of CF containing MWCNTs and ND particles used as nanofillers were observed through scanning electron microscopy (SEM) as shown in Fig.3. The Fig. 3(a) represents the pure CF while $\mathbf{3}$ (b) shows CF foam containing both nanofillers ${ }^{[21-23]}$. As can be seen some very pronounced and significant changes are noticed in the microstructure and porous morphology of CF after the addition of nanofillers. It is also observed that porosity and pore size of CFs become more regular and organized as compared to pure CF (a). Fig. $\mathbf{3}$ ( $\mathbf{c}, \mathbf{d}$ and $\mathbf{f}$ ) reveals the consistent dispersion of ND particles in CF. This further reduces the concentration and viscosity of pretreated pitches during foaming process, indicating the improvement in microstructure of CF. Similarly, Fig.3 (e) corresponds with the more clear description of SEM result of CF containing MWCNTS and NDs ${ }^{[24-26]}$. It can be clearly seen that both nanofillers exist and appear in the SEM measurement. Furthermore, the SEM microstructures confirm that the nanofillers incorporation in CF matrix improves and regularizes the porous morphology of CF significantly.

\subsection{EDX measurements}

In order to confirm the formations of CF composites with MWCNTs and NDs as well as their elemental composition, EDX was performed. During EDX analysis different areas of the nanocomposites were studied and the corresponding peaks are shown in Fig 4 (a) and (b). The EDX spectra presented in Fig.4 (a) reflects that CF/MWCNT composites contain C, $\mathrm{O}$, and $\mathrm{S}$. The presence of $\mathrm{S}$ signals in the spectrum indicates the presence of impurities which came during the synthesis of CF/MWCNTs composite. The EDX spectra in Fig.4 (b) indicates the CF/MWCNTs-NDs composite exhibits $\mathrm{C}, \mathrm{O}, \mathrm{N}$ and $\mathrm{Cr}$. The signal of $\mathrm{N}$ indicates the presence of amino functional group in CF/MWCNTs-ND composite whereas the $\mathrm{Cr}$ signals may be due to some impurities during the synthesis of nanocomposites ${ }^{[27-28]}$. Details of these measured EDX spectra in atomic and weight \% are listed in the attached tables with each Figures.

\section{FTIR observations}

FTIR analysis was performed to confirm the presence of different functional groups on the surface of nanodiamonds. The FTIR data was correlated to the transmittance against wave number and the corresponding vibrational peaks in the range from $500 \mathrm{~cm}^{-1}$ to $4000 \mathrm{~cm}^{-1}$ in which major peaks appeared. These peaks are attributed to the presence of various functionalities over the surface of NDs. It can be seen from graph Fig. 5, that pristine NDs show major peaks at 1110, 1629 and $3435 \mathrm{~cm}^{-1}$, corresponding to the stretching frequencies of $\mathrm{C}-\mathrm{O}, \mathrm{C}=\mathrm{C}$ and $\mathrm{O}-\mathrm{H}$ functions respectively. However additional peak at 3435 
$\mathrm{cm}^{-1}$ constitutes the contribution of $\mathrm{OH}$ group which has been added by the associated impurities ${ }^{[17-30]}$. In case of functionalized NDs there is peaks at 1110, 1382, 1400, $1629 \mathrm{~cm}^{-1}$ predicting the presence of C$\mathrm{O}, \mathrm{COO}, \mathrm{C}=\mathrm{N}, \mathrm{C}=\mathrm{C}$ functions. Beside this, the peak observed at $3350 \mathrm{~cm}^{-1}$ obviously indicates the presence of $\mathrm{N}-\mathrm{H}$ on the surface of functionalized NDs ${ }^{[23]}$. These peaks reveal the functionalization of ball milling system and also its effectiveness in placing the amino functional group on the surface of ND particles.

\section{Physical properties of CF composites}

The effects of additive amounts on the physical properties of CF composites were studies in terms of bulk density, porosity and open cell percentages. Fig. 6 (a),(b) and (c) represent the bulk density, porosity and open cell percentages of CF/MWCNTs and CF/MWCNTs-ND composites. Herein, S1, S2, S3 and S4 reveals blank sample (pure CF with no nanofiller), 1, 2 and 4 wt.\% CF/MWCNTs composites, while S5, S6 and 57 show 1, 2 and 4 wt.\% CF/MWCNTs-ND composites respectively. Fig. 6 (a) shows that the bulk density of $\mathrm{S} 1$ is only $0.72 \mathrm{~g} / \mathrm{cm}^{3}$. When the additive amount of MWCNTs and MWCNTs-ND varies from 1 to $4 \mathrm{wt}$. \%, no remarkable changes in bulk density are observed ${ }^{[31-32]}$. Furthermore, Fig.6 (b) and (c) reveals that porosity and open cell percentages are improved significantly with the incorporation of additive loadings. Fig. 6 (b) shows that the porosity increases from 76 to $78.5 \%$ whenever the additive amount of the MWCNTs and both MWCNTs-NDs are increased in the various samples of CF composites, but the effects of NDs are magnificent as can be clearly seen from the figure. Similarly, the values of the percentage of open cell of CF composites in Fig. 6 (c) increase with the addition of various amount of nanofillers and the highest values are observed for the open cells of CF composites having maximum loadings of hybrid nanofillers. We noticed that all these physical parameters are improved when ND particles are hybrid and synergistically applied with MWCNTs due to which fabrication of reinforcements in matrix is uniformed and reinforcement agglomeration are minimized ${ }^{\text {[15-32] }}$

\section{Mechanical properties of CF composites}

The Mechanical properties of CF composites containing MWCNTs and ND as additives were observed in provisions of compressive strength. The compressive strength of composite containing CF/MWCNTs and CF/MWCNTs-ND among different samples are shown in Fig.7. It is observed that the compressive strength of the CF enhances after the composite formation with different amounts of MWCNTs and ND particles. Fig 7 indicates that pure CF presents a compressive strength of $6.2 \mathrm{Mpa}$. As the amount of MWCNTs is increased from 1 to $4 \mathrm{wt} \%$, the compressive strength increases up to $11.8 \mathrm{MPa}$ for $2 \mathrm{wt} . \%$ MWCNTs in sample. However, further increase in the amount of MWCNTs decreases the compressive strength due to cluster of MWCNTs and poor dispersion with CF matrix ${ }^{[32]}$.Fig.7 also indicates that highest compressive strength is observed when both the MWCNTs and ND particles are synergistically applied in CF matrix. This indicates that the addition of MWCNTs and ND particles significantly enhances the compressive strength. The highest compressive strength of $15.8 \mathrm{MPa}$ is observed at $4 \mathrm{wt} \% \mathrm{MWCNT}-$ ND loadings, which is due to the excellent and uniform dispersion of NDs along with MWCNTs in CF matrix. Fig. 7 also reveals that the compressive strength is considerably improved with the addition of 
different amounts of ND particles among the samples. The reason is that homogeneous dispersion of ND particles among the sample matrix of $\mathrm{CF}$ acts as a dispersion catalyst ${ }^{[10]}$. This indicates better interaction between nanofiller and CF matrix.

\section{Electrical conductivity of CF composites}

Fig.8 indicates the relationship between electrical conductivity of CF with different additives amount of MWCNTs and ND particles. It has been investigated that the electrical conductivity of CF increases with the increase in additive amount of MWCNTs as well as ND particles. It can be seen from Fig. 8 that the electrical conductivity of pure CF is $7.1 \times 10^{3} \mathrm{~S} / \mathrm{m}$. As the amount of MWCNTs increases from 1 to $4 \mathrm{wt} \%$, the electrical conductivity of CF reaches $22.4 \times 10^{3} \mathrm{~S} / \mathrm{m}$ which is much greater than that of pure CF. This enhancement is due to the increase in conduction path of electrons ${ }^{[1-15]}$. Fig.8 also reveals that the electrical conductivity is further enhanced when the samples containing both MWCNTs and ND particles and a maximum electrical conductivity of $30.2 \times 10^{3} \mathrm{~S} / \mathrm{m}$ is observed for $4 \mathrm{wt}$. \% loading of CF/MWCNTsND composite. This is due to uniform and homogeneous dispersion of ND particles between MWCNTs and CF matrix. This study indicates that the consolidation of ND particles with MWCNTs has better effect on the electrical conductivity of CF composites ${ }^{[32]}$.

\section{Thermal Properties of CF Composites}

The thermal conductivity of CF comprising of different additive has been measured from 0 to $800{ }^{\circ} \mathrm{C}$ as shown in Fig.9. It can be seen that CF exhibits thermal conductivity higher than $20 \mathrm{Wm}^{-1} \mathrm{k}^{-1}$, because of graphitic like configuration ${ }^{[10]}$. Fig.9 indicates that the thermal conductivity of $\mathrm{CF}$ is enhanced with the increment of additive loadings of MWCNTs from $0 \mathrm{wt} \%$ (pure CF) to $2 \mathrm{wt} \%$ between 0 to $800{ }^{\circ} \mathrm{C}$. The maximum thermal conductivity of CF containing $2 \mathrm{wt}$ \% is $30.6 \mathrm{Wm}^{-1} \mathrm{k}^{-1}$ which is greater than that of pure $\mathrm{CF}$ at $800^{\circ} \mathrm{C}$. However, 4 wt. \% CF reinforced MWCNT exhibits poor thermal conductivity of $25.9 \mathrm{Wm}^{-}$

${ }^{1} \mathrm{k}^{-1}$ due to the poor and non-uniform dispersion of MWCNT in CF matrix. Fig.9 also reveals that when ND particles are dispersed in CF along with MWCNTs, the thermal conductivity is significantly enhanced with the increase in amount of ND particles. The maximum thermal conductivity of $32.2 \mathrm{Wm}^{-1} \mathrm{k}^{-1}$ is manifested by $2 \mathrm{wt}$ \% CF/MWCNTs-NDs composite. This is due to the consistent dispersion of ND particles between CF sample matrixes along with MWCNTs. From these results, it can be observed that the presence of MWCNTs-NDs hybrid additives considerably enhances the thermal properties of CF matrices.

\section{Conclusion}

The presence of MWCNTs and MWCNTs-ND particles plays a vital role for the improvement of microstructure and pore morphology of CF composites. Carbon foam coupled multi-wall carbon nanotubes (MWCNTs) and CF/MWCNTs coupled nanodiamond hybrid composites were prepared by the direct treatment of MWCNTs and NDs with pretreatment pitches through foaming, graphitization, and 
carbonization process. The results revealed that maximum compressive strength $15.8 \mathrm{MPa}$ and electrical conductivity $30.2 \times 10^{3} \mathrm{~S} / \mathrm{m}$ of CFs were obtained at $4 \mathrm{wt} . \%$ MWCNTs and ND loadings respectively while the maximum thermal conductivity value of $32.2 \mathrm{Wm}^{-1} \mathrm{k}^{-1}$ at $800^{\circ} \mathrm{C}$ was revealed by CFs having $2 \mathrm{wt} . \%$ MWCNTs and NDs loadings.

\section{Declarations}

\section{Deceleration of Interest Statement}

On behalf of all authors, the corresponding author declared that this work is the original work of authors and all standards were followed accordingly and there is no conflict of interest

Dr. Muhammad Khan (Corresponding Author)

\section{Acknowledgments}

This research has been Financially Supported for the Postdoctoral study by the Shaanxi Province and Natural Science Foundation of China under the (Grant No: 2016JQ5108). We are also very thankful to the School of Materials Science and Engineering, Northwestern Polytechnical University, Xian, P.R of China and Higher Education Commission (HEC) Pakistan for their financial support.

\section{References}

1. Chong Chen, Elliot B. Kennel Alfred H. Stiller Peter G. Stansberry, John W. Zondlo, Carbon foam derived from various precursors. Carbon, 2006. 44(8): 1535-1543.

2. J. Marx, A. Brouschkin, S. Roth, D. Smazna, Y. K. Mishra, H. Wittich, K. Schulte, R. Adelung, B. Fiedler, Fundamentals of the temperature-dependent electrical conductivity of a 3D carbon foamAerographite. Synthetic Metals, 2018. 235: 145-152.

3. Kim, J.-H., E. Jeong, and Y.-S. Lee, Characteristics of fluorinated CNTs added carbon foams. Applied Surface Science, 2016. 360: p. 1009-1015.

4. B. Tsyntsarski, B. Petrova, T. Budinova, N. Petrov, A. Popova, M. Krzesinska, S. Pusz, J. Majewska, Synthesis and characterization of carbon foam by low pressure foaming process using $\mathrm{H}_{2} \mathrm{SO}_{4}$ modified pitch as precursor. Bulgarian Chemical Communi-cations, 2009. 41(4): 397-403.

5. Fawcett, W. and D.K. Shetty, Effects of carbon nanofibers on cell morphology, thermal conductivity and crush strength of carbon foam. Carbon, 2010. 48(1): 68-80.

6. Kırca, M, Ayşenur Gül, E. Ekinci, Ferhat Yardım, Ata Mugan, Computational modeling of micro-cellular carbon foams. Finite Elements in Analysis and design, 2007. 44(1-2): 45-52.

7. Seung A Song, Youngmin Lee, Yeon Su Kim, Seong Su Kimet, Mechanical and thermal properties of carbon foam derived from phenolic foam reinforced with composite particles. Composite Structures, 2017. 173: 1-8. 
8. Inagaki, M., J. Qiu, and Q. Guo, Carbon foam: Preparation and application. Carbon, 2015. 87: 128152.

9. Wu, X., Liu, Y., Fang, M., Mei, L., and Luo, B., Preparation and characterization of carbon foams derived from aluminosilicate and phenolic resin. Carbon, 2011. 49(5): 1782-1786.

10. Thomas Beechem, Khalid Lafdi, Ahmed Elgafy, Bubble growth mechanism in carbon foams, Carbon, 2005, 43(5):1055-1064.

11. Sizhong Li, Yongzhong Song, Yan Song, Jingli Shi, Lang Liu, Xinghai Wei, Quangui Guo, Carbon foams with high compressive strength derived from mixtures of mesocarbon microbeads and mesophase pitch, Carbon, 2007, 45 (10): 2092-2097.

12. Zhang, L. and J. Ma, Processing and characterization of syntactic carbon foams containing hollow carbon microspheres. Carbon, 2009. 47(6): 1451-1456.

13. Dodi Irwandi, Zainal Alim Masud, Komar Sutriah, Muhammad Khotib, Preparation and Characterization of Carbon Foam Derived from Fine Coal and Phenolic Resin. Indonesian Journal of Chemistry, 2016. 16(3): 243-248.

14. Hejun Li ,Bin Wang,Yulei Zhang,Yunyu Li,Manhong Hu,Jingxian Xu, Synthesis of carbon nanofibers from carbon foam composites via oxyacetylene torch ablation. Materials and Manufacturing Processes, 2015. 30(1): 54-58.

15. Qilang Lin, Lijuan Qu, Bei Luo, Changqing Fang, Kun LuoLin, , Preparation and properties of multiwall carbon nanotubes/carbon foam composites. Journal of analytical and applied pyrolysis, 2014. 105: 177-182.

16. R. Luo, Y. Ni, J. Li, C. Yang, S. Wang, The mechanical and thermal insulating properties of resinderived carbon foams reinforced by K2Ti6013 whiskers. Materials Science and Engineering: A, 2011. 528(4-5): 2023-2027.

17. Heguang Liu, Yadong Xu, Chaolong Tang, Yuan Li, Nitin Chopra, SiO2 aerogel-embedded carbon foam composite with Co-Enhanced thermal insulation and mechanical properties. Ceramics International, 2019. 45(17): 23393-23398.

18. Muhammad Khan, Li Tiehu, Aqeel A. Khurram, Ting Kai Zhao, Chuanyin Xiong, Zafar Ali, Turab Ali Abbas, Asmatullah, Iftikhar Ahmad, Amjad L. Lone, Sundas Iqbal, Asghar Khan, Active sites determination and De-aggregation of detonation nanodiamond particles. Chiang Mai J. Sci., 2017. 44(3): 1113-1126.

19. Li, Jiantong; Zhang, Guangcheng; Zhang, Hongming; Fan, Xun; Zhou, Lisheng; Shang, Zhengyang; Shi, Xuetao, Electrical conductivity and electromagnetic interference shielding of epoxy nanocomposite foams containing functionalized multi-wall carbon nanotubes. Applied Surface Science, 2018. 428: 7-16.

20. Y.J. Zhai, Z.C. Wang, W. Huang, J.J. Huang, Y.Y. Wang, Y.Q. Zhao, Improved mechanical properties of epoxy reinforced by low content nanodiamond powder. Materials Science and Engineering: A, 2011. 528(24): 7295-7300. 
21. Shameel Farhan, Rumin Wang, Hao Jiang, Kezhi Li, Chuang WangFarhan, A novel combination of simple foaming and freeze-drying processes for making carbon foam containing multiwalled carbon nanotubes. Ceramics International, 2016. 42(7): p. 8980-8989.

22. Yasir A. Haleem, Daobin Liu, Wenxing Chen, Changda Wang, Caihao Hong, Zhen Hec, Jianwei Liu, Pin Song, Shuhong Yu, Li Song, Surface functionalization and structure characterizations of nanodiamond and its epoxy based nanocomposites. Composites Part B: Engineering, 2015. 78: 480487.

23. Schmidlin L, Pichot V, Comet M, Josset S, Rabu P and Spitzer D, Identification, quantification and modification of detonation nanodiamond functional groups. Diamond and related materials, 2012. 22: p. 113-117.

24. Gaies, D. and K. Faber, Thermal properties of pitch-derived graphite foam. Carbon, 2002. 40(7): 11371140.

25. E. Bruneton, C. Tallaron, N. Gras-Naulin, A. Cosculluela, Evolution of the structure and mechanical behaviour of a carbon foam at very high temperatures. Carbon, 2002. 40(11): p. 1919-1927.

26. Xinying Wang, Jiming Zhong, Yimin Wang, Mingfang Yu, Mingfang Yu, A study of the properties of carbon foam reinforced by clay. Carbon, 2006. 44(8): 1560-1564.

27. Caicedo, J., H. Ramirez-Malule, and W. Aperador, Mechanical properties evolution in carbon foams obtained from Guadua angustifolia. Diamond and Related Materials, 2020: 107901.

28. Stobinski L, Lesiak B, Kövér L, Tóth J, Biniak S, Trykowski G, Multiwall carbon nanotubes purification and oxidation by nitric acid studied by the FTIR and electron spectroscopy methods. Journal of Alloys and Compounds, 2010. 501(1): 77-84.

29. Muhammad Khan, Aqeel A. Khurram, Li Tiehu, Ting Kai Zhao, Chuanyin Xiong, Zafar Ali, Naveed Ali, Azeem Ullah, Reinforcement effect of acid modified nanodiamond in epoxy matrix for enhanced mechanical and electromagnetic properties. Diamond and Related Materials, 2017. 78: 58-66.

30. Vadym N Mochalin, loannis Neitzel, Bastian J M Etzold, Amy Peterson, Giuseppe Palmese, Yury Gogotsi, Covalent incorporation of aminated nanodiamond into an epoxy polymer network. ACS nano, 2011. 5(9): 7494-7502.

31. Heguang Liu, Tiehu Li, Yachun Shi, Xilin Wang, Jing Lv, Wenjuan Zhang, Effect of different secondary quinoline insoluble content on the cellular structure of carbon foam derived from coal tar pitch. Journal of Analytical and Applied Pyrolysis, 2014. 108: p. 310-315.

32. Heguang Liu, Tiehu Li, Yachun Shi, Xilin Wang, Jing Lv, Effect of multi-walled carbon nanotube additive on the microstructure and properties of pitch-derived carbon foams. Journal of materials science, 2015. 50(23): 7583-7590.

\section{Figures}



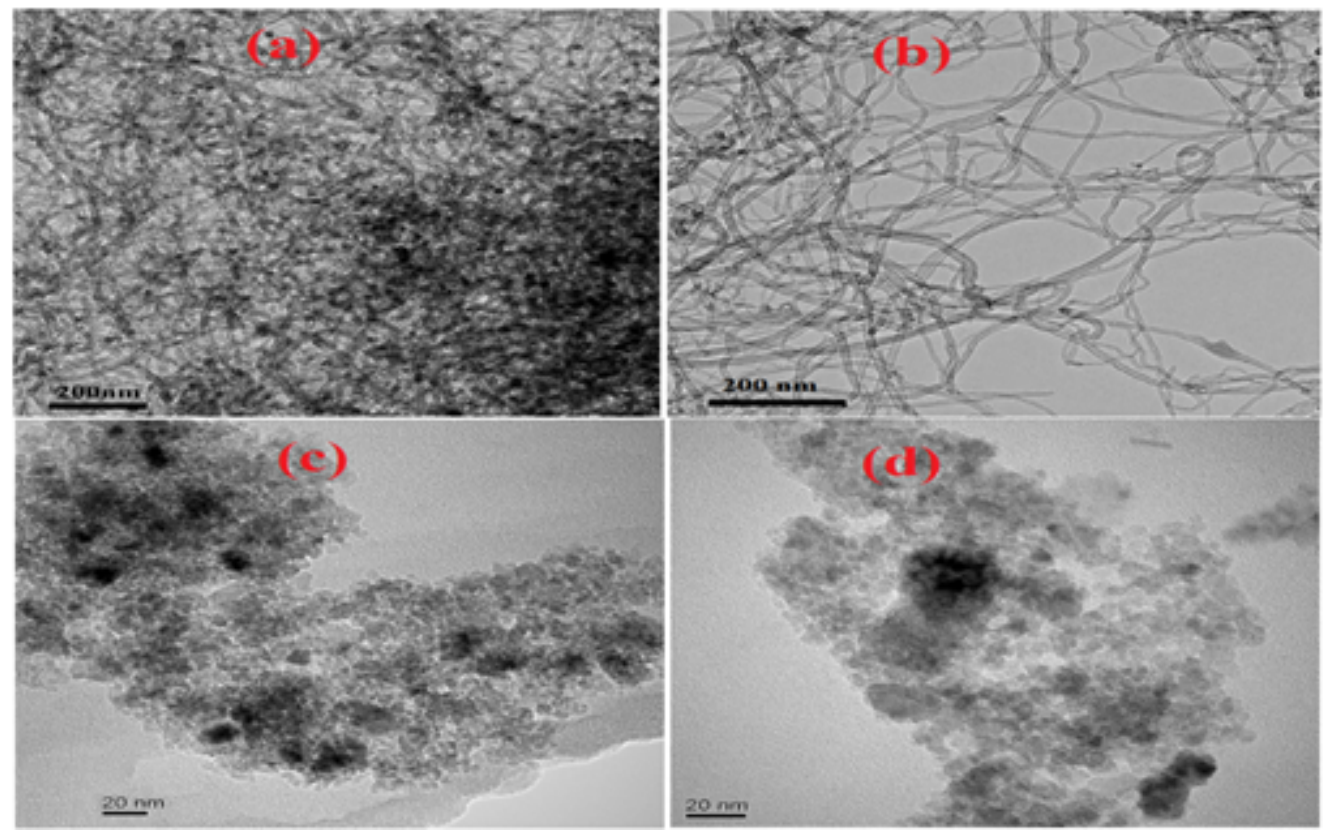

\section{Figure 1}

TEM images of (a) non-purified MWCNTs, (b) purified MWCNTs, (c) non-functionalized NDs and (d) functionalized ND powder.

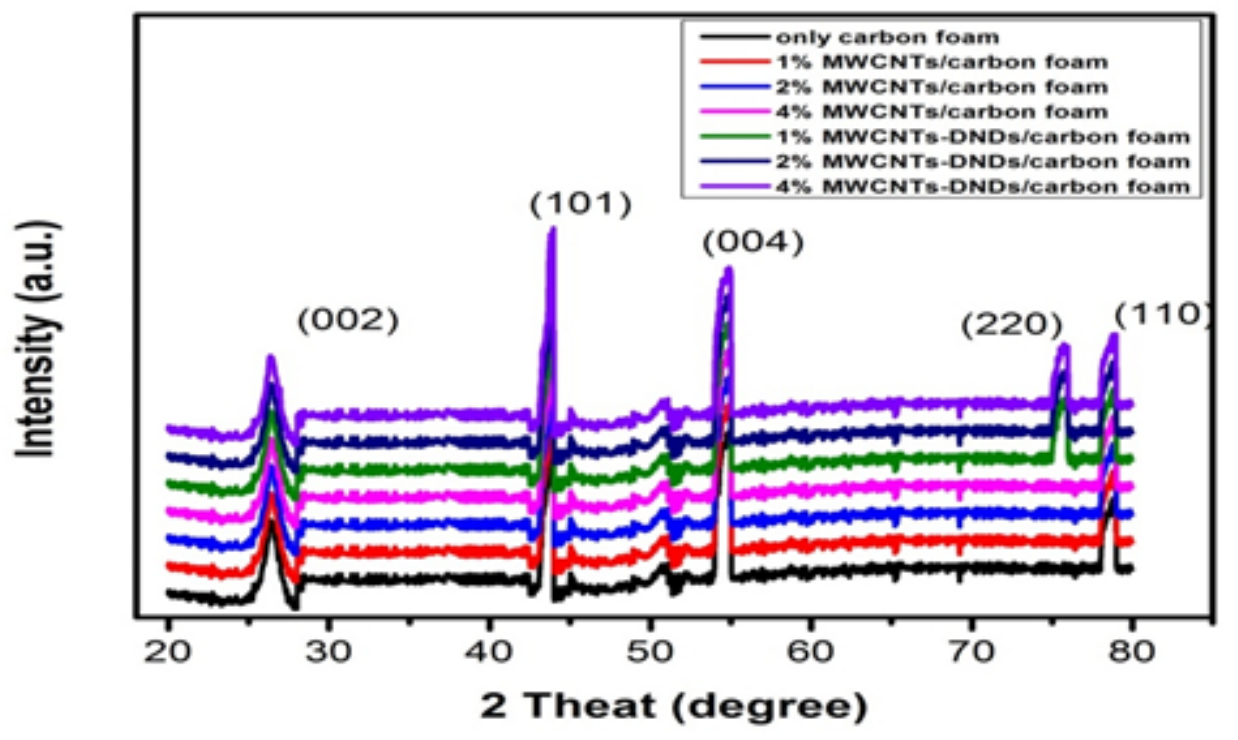

Figure 2

XRD patterns of carbon foam possessing various additive loadings of MWCNTs and NDs. 

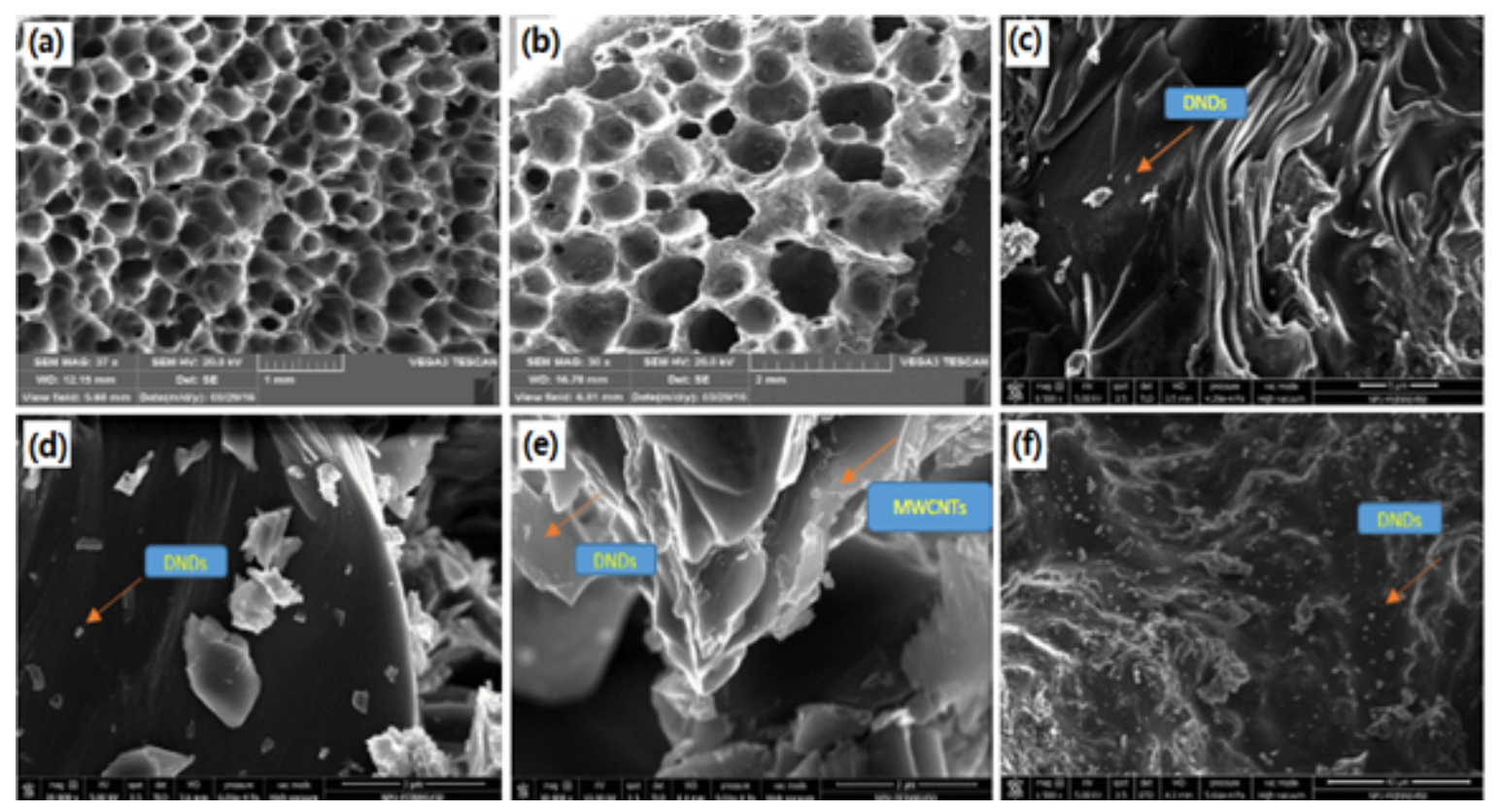

Figure 3

SEM results of CF composites having MWCNTs and NDs as nanofillers. 

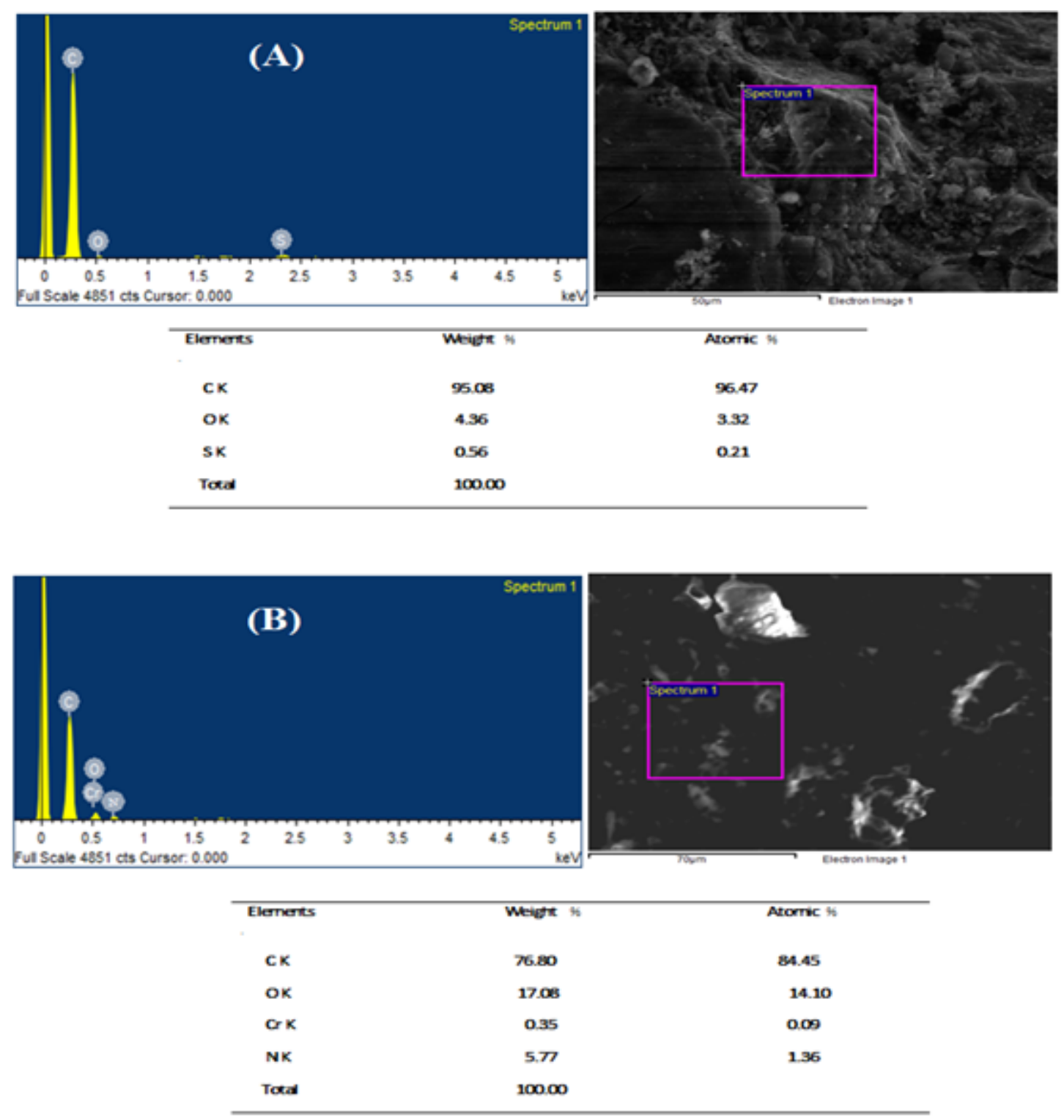

Figure 4

EDX observations of (a) CF/MWCNTs and (b) CF/MWCNTs-ND composites.

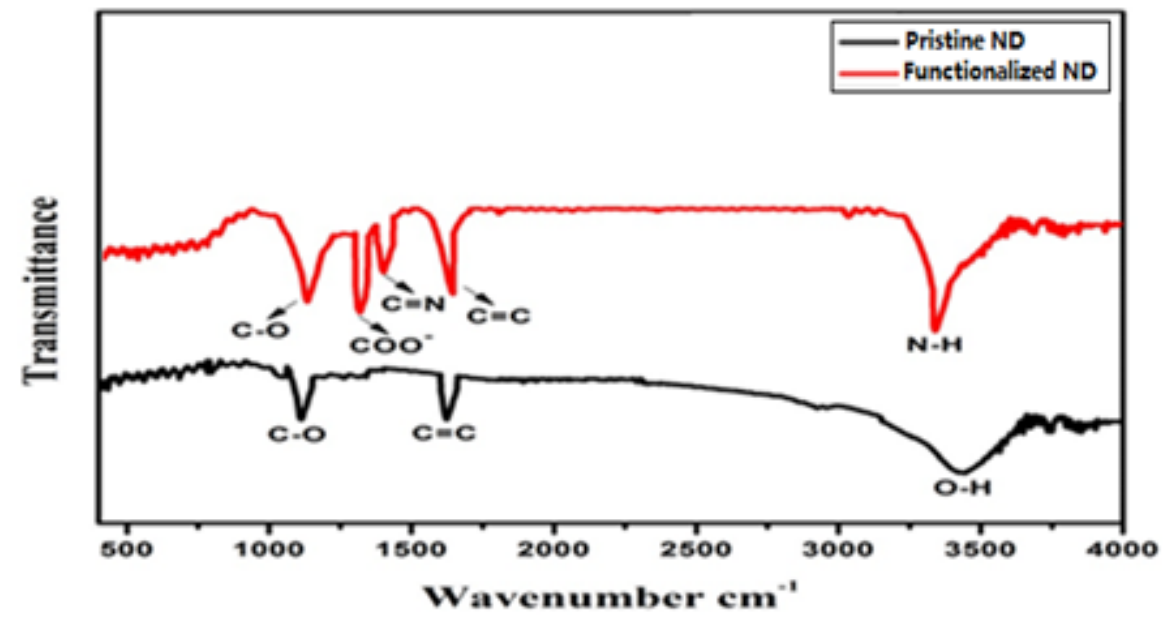

Figure 5 
FTIR measurements of pristine and functionalized NDs.
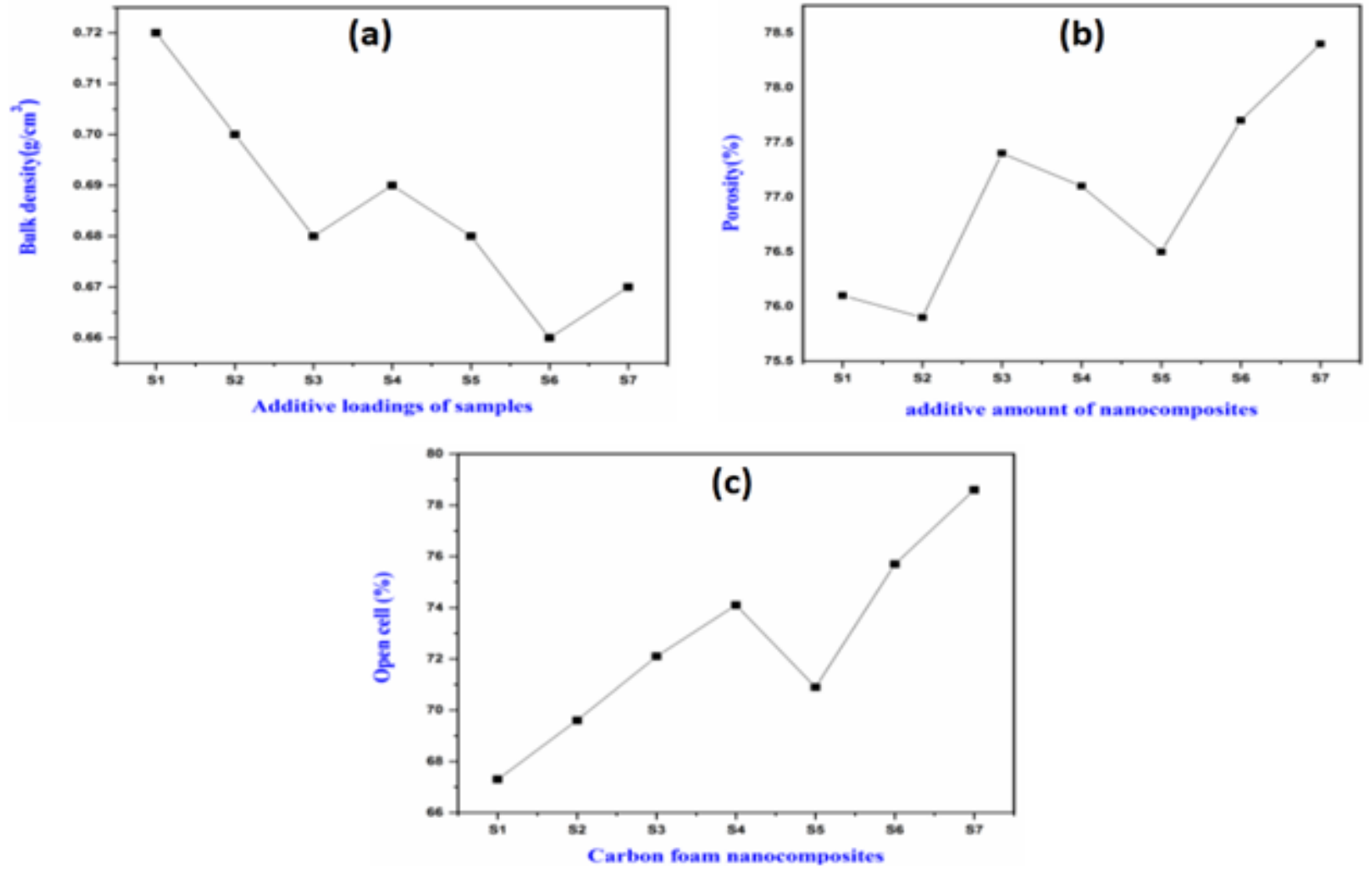

Figure 6

bulk density relationship of CF composites with respect to nanofillers loadings.

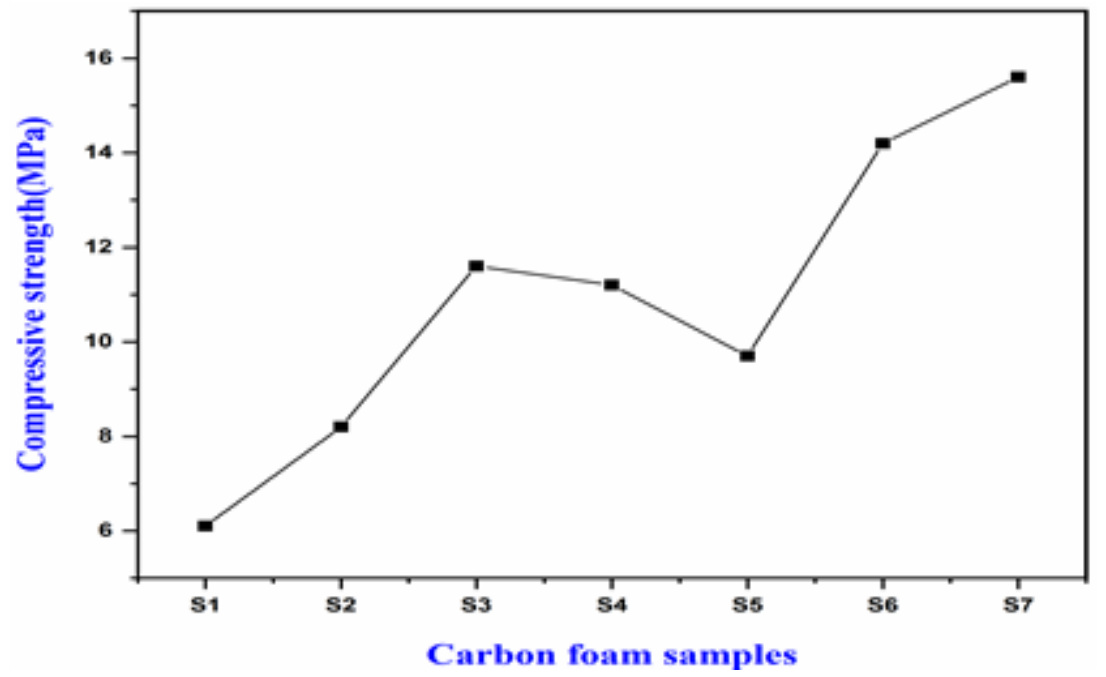

Figure 7

Compressive strength of CF composites containing variable amounts of additives. 


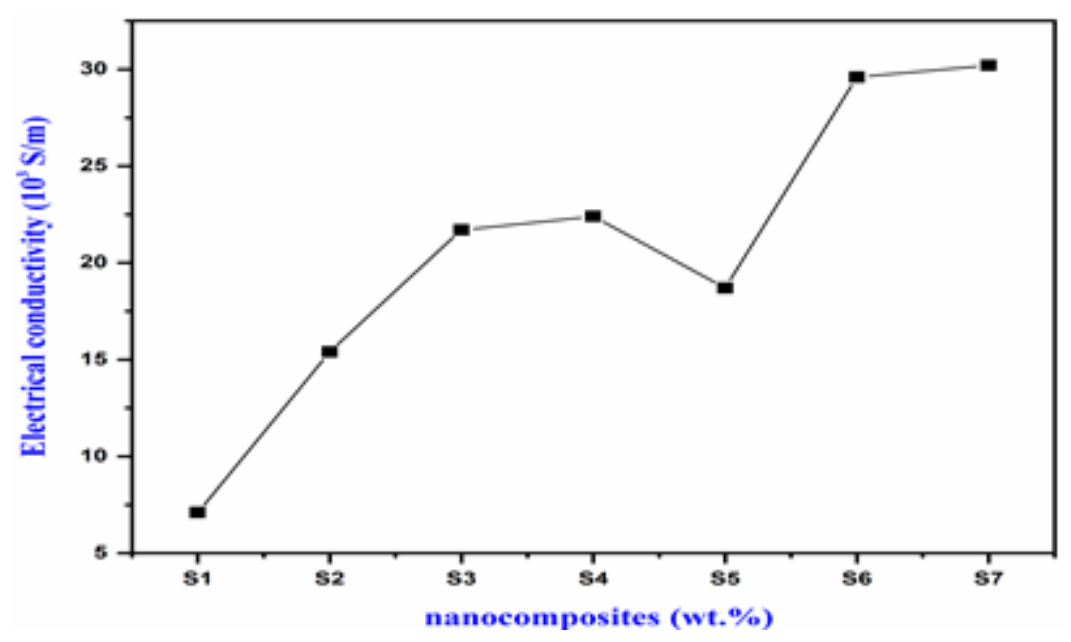

Figure 8

electrical conductivity of CF composites having different amount of additives.

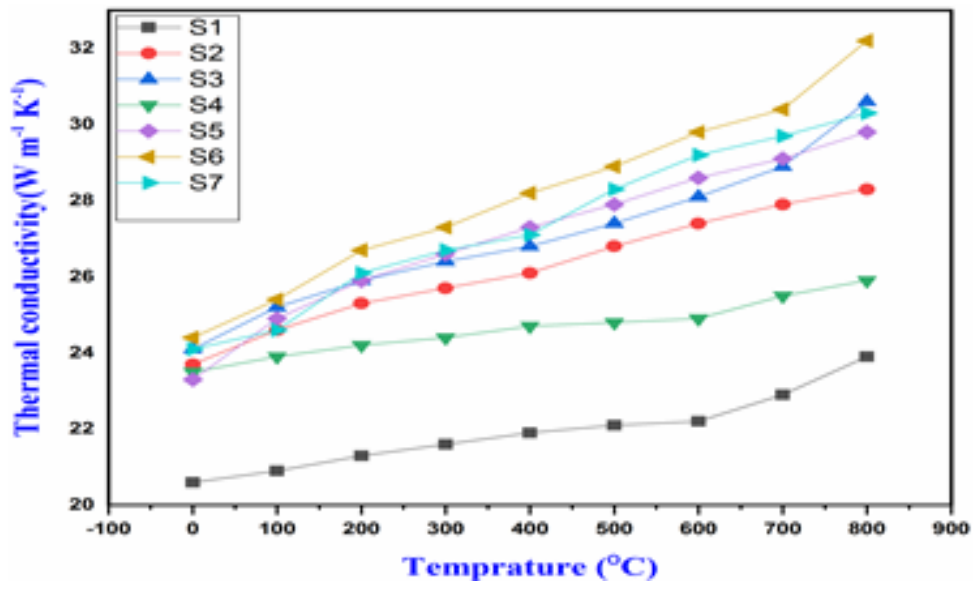

Figure 9

Thermal conductivity of CF samples containing various amounts of additives.

\section{Supplementary Files}

This is a list of supplementary files associated with this preprint. Click to download.

- supplementarymaterials.docx 